\title{
Science and Health for All Children with Cancer
}

Catherine G. Lam ${ }^{1,2}$, Scott C. Howard ${ }^{2,3,4}$, Eric Bouffet ${ }^{4,5}$, Kathy Pritchard-Jones ${ }^{4,6}$

${ }^{1}$ Departments of Global Pediatric Medicine and Oncology, St. Jude Children's Research Hospital, Memphis, TN, USA

${ }^{2}$ University of Tennessee Health Sciences Center, Memphis, TN, USA

${ }^{3}$ Guardian Research Network, Spartanburg, SC, USA

${ }^{4}$ Board, International Society of Paediatric Oncology (SIOP), Zug, Switzerland

${ }^{5}$ Division of Haematology/Oncology, Hospital for Sick Children, University of Toronto, Toronto, ON, Canada

${ }^{6}$ University College London, UCL Great Ormond St Institute of Child Health, London, UK

\begin{abstract}
(137 words)
Each year approximately 429,000 children and adolescents aged 0-19 years are expected to develop cancer. Five-year survival rates exceed $80 \%$ for the 45,000 children with cancer in highincome countries (HIC), but are less than $30 \%$ for the 384,000 children in low- and middle-income countries (LMIC). Improved survival rates in HIC have been achieved through multidisciplinary care and research, with treatment regimens utilizing mostly generic medicines and optimized risk stratification. Children's outcomes in LMIC can be improved by adapting effective treatments to local resources and clinical needs, and addressing common problems such as delayed diagnosis and treatment abandonment. By supporting local leaders to increase service capacity and achieve measurable clinical improvements, collaborative partnerships can stimulate governmental and nongovernmental investments. These approaches should bring the new WHO childhood cancer survival target of $60 \%$ within reach of all by 2030 .
\end{abstract}




\section{Main Text (3,724 words)}

In recent years, 5-year survival rates, typically equating to cure for children with cancer, have risen to about $80 \%$ in most high-income countries (HIC) (1-4). This progress partly reflects optimized use of conventional therapies (e.g., cytotoxic drugs) through better risk stratification of patients. For example, on the basis of molecular prognostic markers, treatments are intensified for patients identified as high-risk and de-escalated for patients identified as lower-risk to reduce the risks of immediate and long-term side effects. Expanding portfolios of new drugs that target the biological mechanisms driving the growth of pediatric cancers are also starting to contribute to improved cure rates in HIC (5). The situation is more bleak for children with cancer in low- and middleincome countries (LMIC) (6). In LMIC taken as a whole, the 5-year survival rate is only about $30 \%$ (Table 1). Even considering only geographic sites with adequate resources to support population-based cancer registration, recent global data show that the 5-year survival rate for children can be up to $45 \%$ higher in HIC than in LMIC for acute lymphoblastic leukemia, and up to $51 \%$ higher for children with brain tumors (1).

In 2018, the World Health Organization (WHO) set a global survival target of $60 \%$ for all children with cancer, with the goal of saving a million more lives by the year 2030 (7). This new WHO target may appear daunting, but cannot be ignored. About $89 \%$ of the world's children (age 0 to 19 years) live in LMIC and they account for $95 \%$ of the mortality from cancer in this age group

worldwide (Table 1) (4, 6). Furthermore, the incidence rates of many childhood cancers are increasing by about $1 \%$ per annum in many countries with population-based cancer registration (8). In this Review, we aim to demonstrate that this target is tractable, building on the longestablished work of the global childhood cancer community. Success will also require new partnerships and increased cooperation between stakeholders, including not only health care professionals, but also parents, patients, civil society communities, industry, academia and governments (9).

\section{Global Incidence of Childhood Cancers}

The major cancer types affecting children younger than 15 years and in adolescents (aged 15-19 years) differ from those affecting adults, which are typically epithelial in origin. The most common 
cancers in children include acute leukemias, brain tumors, lymphomas, bone and soft tissue sarcomas and germ cell tumors. The typical 'embryonal tumors' (neuroblastoma, renal tumors and retinoblastoma) are confined largely to younger children whereas cancers in adolescents include more epithelial tumors (such as thyroid carcinoma) and melanoma. An estimated 429,000 new cases of childhood cancer are expected globally each year (Table 1). Globally, the agestandardized reported incidence rates are 141 per million person-years (children) and 185 per million person-years (adolescents) (8). Age-specific incidence rates vary by geography and ethnicity. Notably, most of the world's children are not covered by population-based cancer registries (10). Furthermore, the 170 per million incidence in HIC is double that of low-income countries (LIC) and lower-middle-income countries (LMIC) (Table 1, Fig. 1). However, if one extrapolates incidence from HIC to the sub-Saharan population, it is apparent that less than half the expected cases of acute leukemia, brain tumors, neuroblastoma and bone tumors are diagnosed (Fig. 1). In contrast, there is less of an incidence deficit for cancers that present with more obvious clinical symptoms such as Burkitt lymphoma, renal (Wilms) tumor and retinoblastoma (3). Similar conclusions can be drawn when comparing incidence rates in India with those in Japan and Korea (8).

Within each of the main cancer types, there are subcategories that can be treated with generic medicines and regimens that are readily adapted to resource-limited settings. These include Burkitt lymphoma, Wilms tumor, acute lymphoblastic leukaemia, Hodgkin lymphoma, certain brain tumors, germ cell tumors, and low-risk or localized cases of neuroblastoma, sarcomas and retinoblastoma. Approximately 50\% of all cancers in the 0-19 years age group would be in this "favorable prognosis" category if diagnosed and treated appropriately.

Unfortunately, treatment failure is common in LMIC due to many factors, including failure to diagnose, misdiagnosis, unaffordable or abandoned treatment, toxic (treatment-related) death, and excess relapse (Fig. 2) $(9,11,12)$. Delayed presentation or diagnosis, drug shortages, intermittent adherence and treatment regimens that are of reduced intensity to facilitate tolerability also contribute to treatment failure. As discussed below and summarized in Fig. 3, many causes of treatment failure are preventable. While children with cancer can be managed in very different settings in LMIC, we will use the term "cancer center" to characterize dedicated wards/units for 
children with cancer within a hospital, as well as stand-alone hospital facilities for patients (solely for or including children) with cancer.

\section{The challenge of late diagnosis}

Patients whose cancer diagnosis is delayed often present with more advanced disease that is inherently harder to cure and necessitates more intensive therapy. Such patients often have comorbidities, such as malnutrition and infection and are at increased risk of tumor lysis syndrome and treatment-related death $(9,13-19)$. When delayed diagnosis is associated with more refractory disease and excess relapse, this increases the costs and morbidity of treatment, and in turn increases treatment abandonment. As demonstrated in HIC, community-based public and professional awareness-raising campaigns can be effectively coupled with diagnosis-specific referral guidelines; in the UK, such a national strategy reduced the median time to diagnose brain tumors in children from 14 weeks to just under 7 weeks (20). In Honduras, awareness materials on retinoblastoma, the most common eye cancer in young children, were integrated into national immunization platforms across health centers, and resulted in a significant decrease in the proportion of patients presenting with advanced retinoblastoma (21). Other resources to increase

timely diagnosis of childhood cancer have been developed for various settings, including educational resources from the Pan American Health Organization, as well as locally adapted and international Signs and Symptoms Campaigns, with ongoing opportunities to demonstrate effectiveness, especially in LMIC (22-24).

\section{Reducing diagnostic failure}

Cancer diagnosis can be hampered by lack of appropriately trained workforce, imaging and laboratory equipment, and access to more specialized techniques such as immunohistochemistry and immunophenotyping that are routine in HIC pathology departments. These deficits may affect individual treatment centers or entire geographic regions. Even Burkitt lymphoma, which has a characteristic histologic appearance and is commonly diagnosed in Sub-Saharan Africa, has been shown to be misdiagnosed in 18-35\% of cases in single and multi-centre analyses (Fig. 2) (25-27). Discrepancies in pathology diagnosis reached $64 \%$ in one study from Uganda that included all subtypes of suspected non-Hodgkin lymphoma $(26,27)$. In addition to diagnostic difficulties, staging and risk stratification are suboptimal when patients lack access to imaging methods and 
assays that measure prognostic biomarkers (e.g., amplification of the $M Y C N$ oncogene for neuroblastoma) (28). Barriers to access can be due to the unavailability of services or the inability of patients to pay for these services in health care systems that primarily depend on families' outof-pocket contributions (29). Strategies to facilitate earlier and more accurate cancer diagnosis in LMIC include the use of telemedicine with local, cross-regional or international expert groups to complement on-site continuing education; both clinical referral networks and professional networks for local input or virtual input from international experts have been successfully implemented in $\operatorname{LMIC}(3,9,30-35)$. An example of such long-term collaboration is outlined later in this Review.

\section{Targeting toxic (treatment-related) death}

Toxic death is a leading contributor to treatment failure for children with cancer in LMIC $(9,32)$. The occurrence rate can be as high as $24 \%-30 \%$ in higher-risk patients in the first month of therapy, with risk depending on the cancer type, regimen used, and supportive care available (14, 36-38). Appropriate supportive care especially at the start of treatment is one of the biggest challenges in LMIC (39). Nutritional support, management of infections, and aggressive hydration (for hematologic cancers and bulky tumors) to prevent tumor lysis syndrome can effectively reduce early toxic death (40). Resource-sensitive tools and interventions to address patients' nutritional needs in LMIC have been developed as key components of supportive care (41). In some areas of Africa, intestinal parasite therapy is routinely given prior to the start of chemotherapy, to help protect children from common infections that can become overwhelming and potentially fatal when the child's immune system is weakened by malignancy as well as their treatment. Likewise, during treatment, other forms of antimicrobial prophylaxis and therapy have been adapted to localities where the incidence and types of infection differ from those in HIC (17). To help staff respond quickly to infections and other causes of rapid clinical decline in children with cancer, an early warning system has been developed to facilitate identification, team communication and management of children with cancer who are deteriorating in LMIC (42). In collaboration with St. Jude Children's Research Hospital, this early warning system was validated in Guatemala, where the system effectively reduced the number of children who deteriorated, and decreased the need for patients to be transferred to the intensive care unit (43). Strategies to adapt the treatment 
regimen to local resources and needs, with the intent to reduce toxic deaths, are further described below.

\section{Adapting treatment regimens in LMIC}

So-called "adapted treatment regimens" are widely used in LMIC. These are typically lower intensity regimens that can be associated with higher overall survival by causing fewer toxic deaths (44). For instance, patients with Burkitt lymphoma who have bulky disease and significant morbidity after initial treatment may be managed with an additional few weeks of reducedintensity treatment before continuing standard therapy (45). To address the challenges of bed shortages and other treatment delays (potentially compounding patients' risks of not tolerating treatment well as their condition may worsen during the wait), some providers have incorporated inexpensive medicines that can be administered on an outpatient basis (e.g., hydroxyurea for patients with acute myeloid leukemia). Other providers, to mitigate known chemotherapy toxicity in settings with less available supportive care, have studied the use of reduced chemotherapy doses, as well as low-cost modifications to facilitate monitoring when measurement of drug levels is not possible (46, 47, 48Peña-Hernandez, 2019 \#15297Peña-Hernandez, 2019\#15297). Since adapted treatment regimens may also increase the risk of relapse due to reduced intensity treatment, local data for both outcomes (toxic death rate and relapse rate) need to be continuously monitored (49, 50). In LMIC, many factors unrelated to the treatment regimen also increase relapse risk, including unavailability or unaffordability of medicines, lack of adherence to treatment, and lack of infrastructure and support to families that enable treatment completion (Fig. 3) (35, 51-55). Ideally, these factors should be considered in the planning of adapted treatment regimens - for instance, by planning medicines that can be substituted for commonly unavailable or unaffordable medicines, or including funding for a clinical coordinator/patient navigator to help reinforce adherence to the treatment regimen. The International Paediatric Oncology Society (SIOP, Www.SIOP-online.org) has established procedures to develop and deploy such regimens $(28,41$, 56-58).

\section{Reducing treatment abandonment}

Treatment abandonment, defined as 4 weeks or more of missed appointments during therapy, is a major contributor to treatment failure for children with cancer in most cancer centers in LMIC, but 
is rare in $\mathrm{HIC}(12,35,38,46,59-63)$. In some settings such as rural Zambia, abandonment rates approach 50\%; after war or during civil unrest or natural disasters, abandonment rates increase sharply (64). In Côte d'Ivoire, nearly half of the children with Burkitt lymphoma abandoned treatment shortly after the first admission, resulting in only a 6\% cure rate (Fig. 2) (65). Risk factors for abandonment include poverty as well as the local cost of treatment, low educational attainment of parents, distance from the cancer center, cancer type and, in some cases, patient gender; one study showed that the 12-month cumulative incidence of abandonment was $22 \%$ in females versus $7 \%$ in males (13). Government support matters; in a study from Kenya where more than $70 \%$ of children with cancer lacked health insurance at diagnosis, these children had a 3 -fold higher risk of treatment failure (most commonly treatment abandonment) than those with insurance (66). To address treatment abandonment, various strategies have been deployed successfully. In Central America, only $6.5 \%$ of patients with anaplastic large-cell lymphoma abandoned therapy, somewhat lower than the abandonment rate typically experienced with other tumors in the same settings, perhaps because of relatively short treatment duration (compared to acute lymphoblastic leukaemia) and government coverage of all chemotherapy costs (67-69). However, in Sierra Leone, despite shortening the duration of inpatient treatment and the provision of free treatment, meals and transportation, treatment abandonment persisted in rural areas, a finding replicated in other countries with long travel times and no established referral network (38, 61, 70). In El Salvador, in addition to free treatment, implementation of a tracking protocol with community-based interventions for missed appointments successfully prevented abandonment in almost all patients, while in Recife, Brazil, a comprehensive social support and educational program reduced abandonment from $16 \%$ to $0 \%(38,71)$. To further explore local creative strategies to address treatment abandonment and related topics, the global SIOP community has organized active working groups and launched a podcast related to these issues (59).

\section{Stimulating development of comprehensive health services for children with cancer}

Many causes of preventable treatment failure in LMIC noted above are rooted in fragile or insufficient health care infrastructure (including facilities) and workforce. Childhood cancer can provide a lens to examine and improve the performance of health care systems more generally, with potential benefits beyond children with cancer. Management of childhood cancer in LMIC is ideally facilitated by being part of a national cancer control programme that coordinates 
identification of priorities and resource allocation, and supports service delivery including needs for workforce, essential medicines and technologies, as well as information systems and policies (72). Additional work should be done locally to understand and address the root causes of diagnostic, referral and treatment delays and inform data-driven solutions to improve care coordination and optimize resource allocation (73). For example, improvements in the laboratory and pathology infrastructure and in team communication should lead to faster diagnosis and response to patients' critical electrolyte derangements or life-threatening infections - benefitting all patients not only children with cancer. Similarly, prioritization of hand hygiene and isolation rooms for infection prevention and control should decrease morbidity and mortality for children with cancer and all other patients in the same hospital. The design of strategies to improve care should integrate the input of partners across the health system - not only clinicians, but also others such as policy makers responsible for budget allocations, legal and regulatory bodies overseeing approval of medicines, academic bodies overseeing the training and accreditation of providers, and civil society organizations providing support for patients and families. Successful sustained partnerships have stimulated the engagement of other local governmental and nongovernmental partners to invest in services benefiting children (73).

Importantly, national policies should support the ongoing collection and analysis of local data for monitoring and evaluation, starting with allocating resources for infrastructure and trained personnel to register all newly diagnosed patients, and to document core patient outcomes such as survival, abandonment and relapse $(71,74,75)$. Beyond increasing survival alone, coordinated policy efforts for children with cancer can improve patients' quality of life and reduce suffering by ensuring access to morphine for pain as well as palliative care and psychosocial support (76). As more children survive cancer, policies can also stipulate resources to support the distinct needs of children and families affected by a chronic illness like cancer, while strengthening local capacity to prevent, monitor, and manage late effects of treatment amongst survivors (76-78).

\section{Access to essential medicines}

An additional challenge affecting the care of children with cancer in LMIC is access to essential medicines. WHO has provided global guidance recommendations in the form of a Model List of Essential Medicines (EML) across health conditions; these include common cancers for 
adolescents and adults since 1977, and more recently for children (up to age 12) since 2007 (79). Recent analyses suggest an ongoing need for implementation research within countries to facilitate access to the recommended medicines, particularly in resource-limited settings $(54,80)$.

A further complexity is guaranteeing supply of high quality medicines. Here, the experience of Brazil with asparaginase is instructive. An essential medicine for the most common childhood cancer, acute lymphoblastic leukemia, asparaginase was approved by the US Food and Drug Administration in 1978 and recognized in the WHO EML since 1993 (79, 81). However, native asparaginase (derived from the bacterium Escherichia coli) has been only intermittently accessible in many settings (82). In 2017, anticipating drug shortage of native asparaginase, the Ministry of Health in Brazil changed the national supplier to a foreign manufacturer that offered a new lowercost generic product (83). Concerned by the lack of published data on this new product, investigators in Brazil studied the drug's properties, and found that it was less bioactive, and contained contaminating proteins that increased the risk for immune-related side effects compared with the native asparaginase used previously $(83,84)$. Although the nationwide distribution of this new product in Brazil was halted, this drug continues to be manufactured and distributed to other countries around world (83). Moving forward, the WHO's 2018 global initiative will hopefully engage additional partners to increase economies of scale and uphold accountability mechanisms

for product availability and quality consistency worldwide. Lessons learned from addressing social and financial barriers to increase access to medicines could then also be leveraged for other critical technologies (7). High-quality data and research will be critical in directing how resources can be harnessed to reach WHO's global goal.

\section{Science and drug development for all}

Most children with cancer in HIC are enrolled in research and multi-center clinical trials, and this has been described as a key factor contributing to the increased survival rates seen $(2,4,85)$. However, there are several obstacles to further improvements in survival rates: (i) pediatric cancer is rare; (ii) the histologic subtypes of pediatric cancers differ substantially from those in adults; and (iii) the high cure rate for many pediatric cancers in HIC means that only small numbers of children with relapsed or refractory disease are available to enrol in early-phase trials of new drugs. Hence, effective drug development in pediatric oncology can benefit from engaging LMIC 
partners. Anti-CD20 antibody therapies are one example. Non-Hodgkin lymphoma (NHL) affects 70,000 adults each year in the USA and several anti-CD20 antibodies are available or in development for CD20-positive disease $(86,87)$. However, in the USA only 400 children are diagnosed annually with CD20-positive NHL across more than 200 pediatric oncology centers; fewer than 80 relapse, and even fewer relapse a second time and would be eligible for clinical trials for refractory mature B-cell NHL, which means that pediatric trials of novel CD20 antibodies are limited to a small number of eligible patients treated in a large number of centers $(8)$. Furthermore, under the current paradigm, multiple pharmaceutical companies may compete for the same patient population to test different products with the same mechanism of action. It is not surprising that many drug development programs fail despite prolonged and costly efforts to meet accrual targets.

To address these issues in a HIC setting, the Accelerate Platform was launched in Europe and has been expanded to include partners worldwide (https://www.accelerate-platform.eu/about-us/). Strategic aims include ensuring that drug development for children and adolescent cancers be based on pharmaceutical mechanism of action rather than adult indications alone, and facilitating international collaboration between all stakeholders - including researchers, pharma and governmental regulators in conjunction with influential parent/patient advocates and resourcing partners (88-90). While currently only European and North American centres are involved, there is the potential to involve well-structured cancer centres from LMIC, and indeed this may be essential for timely progress. Comparative analysis of survival rates between HIC and LMIC are limited by many factors, including different proportions of high-risk patients and environments of care, thus continuous research in LMIC remains vital to improve outcomes $(14,37,91)$. Furthermore, only $11 \%$ of children with cancer live in HIC, so there is an untapped opportunity to help the remaining $89 \%$ of children who develop cancer in LMIC by providing them access to novel therapies through clinical trials, while accelerating scientific progress that can benefit all children (8).

The "twinning program" between the pediatric oncology/hematology department at King Hussein Cancer Center (KHCC) in Amman (Jordan) and the neuro-oncology section at the Hospital for Sick Children, Toronto (Canada) exemplifies the benefit of collaborative research between institutions in LMIC and HIC to advance science as well as patient outcomes. Stemming from 
collaborations since 2004, monthly multidisciplinary video-teleconferences were held to discuss the management of KHCC patients. On average 4 to 5 patients were discussed during each 1-hour session. The benefits of this interaction quickly became evident for all participants (92-96). The group conducted a series of research projects together, and found that 17 of 44 (39\%) children with high-grade malignant brain tumour cases at KHCC over 10 years had defective mismatch repair (MMR) genes, compared to less than $4 \%$ in North America $(97,98)$. This research led to the development of a larger collaborative network, the MMRD consortium, which in turn facilitated analyses of tumor and blood samples from individuals with MMRD-associated tumors, which are ultra-hypermutated and responsive to immune checkpoint inhibitors. Indeed, sustained remissions have been reported in MMRD patients treated with the checkpoint inhibitors nivolumab and ipilimumab $(99,100)$, and continued efforts are now warranted to initiate immunotherapy trials and studies of adapted treatment regimens in LMIC. Clinical trials involving LMIC partners can integrate local epidemiology and resource considerations into the trial design, and address new implementation research questions while galvanizing partnerships to sustain progress $(34,101$ 104).

\section{Conclusion}

Childhood cancer demonstrates how a relatively small group of stakeholders investing in a relatively uncommon cluster of diseases has the potential to provide systemic benefits for science and health. As we have discussed, nearly $40 \%$ of the world's children expected to have cancer are undiagnosed. We need to address this problem of children not being diagnosed, while continuing to improve treatment for those diagnosed. We need to invest in more prospective research and clinical trials to improve care for children in LMIC, with the expectation that lessons learned can translate to global improvements. Achieving health, defined by WHO as "not merely the absence

of disease," requires cross-cutting investments to address underlying determinants of health and needs across the health system $(105,106)$. We advocate for health for all children with cancer, as summarized in the Erice Statement: "the long-term goal of the cure and care of a child with cancer is that he/she becomes a resilient and autonomous adult with optimal health-related quality of life, accepted in society at the same level as his/her peers" (107). There is no health for all unless there is also science for all. 
Table 1. Estimated numbers of pediatric cancer patients each year by country and World Bank

\begin{tabular}{|l|c|c|c|c|c|c|c|c|}
\hline $\begin{array}{l}\text { World } \\
\text { bank } \\
\text { income } \\
\text { group }\end{array}$ & $\begin{array}{c}\text { Population } \\
\text { o to 19 } \\
\text { years old } \\
\text { (millions) }\end{array}$ & $\begin{array}{c}\text { Mean cancer } \\
\text { incidence per } \\
\text { million } \\
\text { children }\end{array}$ & $\begin{array}{c}\text { Expected } \\
\text { cancer } \\
\text { incidence } \\
\text { per million } \\
\text { children }\end{array}$ & $\begin{array}{c}\text { Children } \\
\text { with cancer } \\
\text { reported } \\
\text { annually }\end{array}$ & $\begin{array}{c}\text { Children } \\
\text { with cancer } \\
\text { expected } \\
\text { annually* }\end{array}$ & $\begin{array}{c}\text { Estimated } \\
\text { children } \\
\text { cured }\end{array}$ & $\begin{array}{c}\text { Cure } \\
\text { rate of } \\
\text { treated } \\
\text { children }\end{array}$ & $\begin{array}{c}\text { Cure rate } \\
\text { of all } \\
\text { expected } \\
\text { cancer } \\
\text { cases }\end{array}$ \\
\hline LIC & 345 & 83 & 170 & 28635 & 58650 & 5865 & $20 \%$ & $10 \%$ \\
\hline LMIC & 1205 & 86 & 170 & 103630 & 204850 & 40970 & $40 \%$ & $20 \%$ \\
\hline UMIC & 707 & 124 & 170 & 87668 & 120190 & 54086 & $62 \%$ & $45 \%$ \\
\hline HIC & 266 & 170 & 170 & 45220 & 45220 & 36176 & $80 \%$ & $80 \%$ \\
\hline Total & 2523 & 105 & 170 & 265153 & 428910 & 137097 & $52 \%$ & $32 \%$ \\
\hline
\end{tabular}

LIC, low-income countries; LMIC, lower-middle-income countries; UMIC, upper-middle-income countries; HIC, high-income countries; LMIC, low- and middle-income countries.

* The expected number of cancer cases per year (170/million) is calculated by applying the mean agespecific annual incidence rates of all high-income countries from Globocan

(http://gco.iarc.fr/today/online-analysis-table) to the population of each country $(8,108)$. The incidences in each pediatric age cohort are 200/million among 0 to 4 year olds, 135/million among 5 to 9 year olds, 135/million among among 10 to 14 year olds, and 211/million among 15 to 19 year olds. The United Nations population numbers were retrieved February 3, 2019 (109).

** Calculated by adding all 4 World Bank income groups together.

+ The incidence gap is defined as the percentage of patients expected to develop cancer each year divided by the mean of the incidence reported in high-income countries. Some variation is expected based on epidemiologic variation; whereas, lack of diagnosis is a likely cause of gaps that exceed 2 standard deviations from the high-income country mean (108). 
Fig. 1 Childhood cancer Incidence deficit: Percentage of expected cancer cases diagnosed in each country

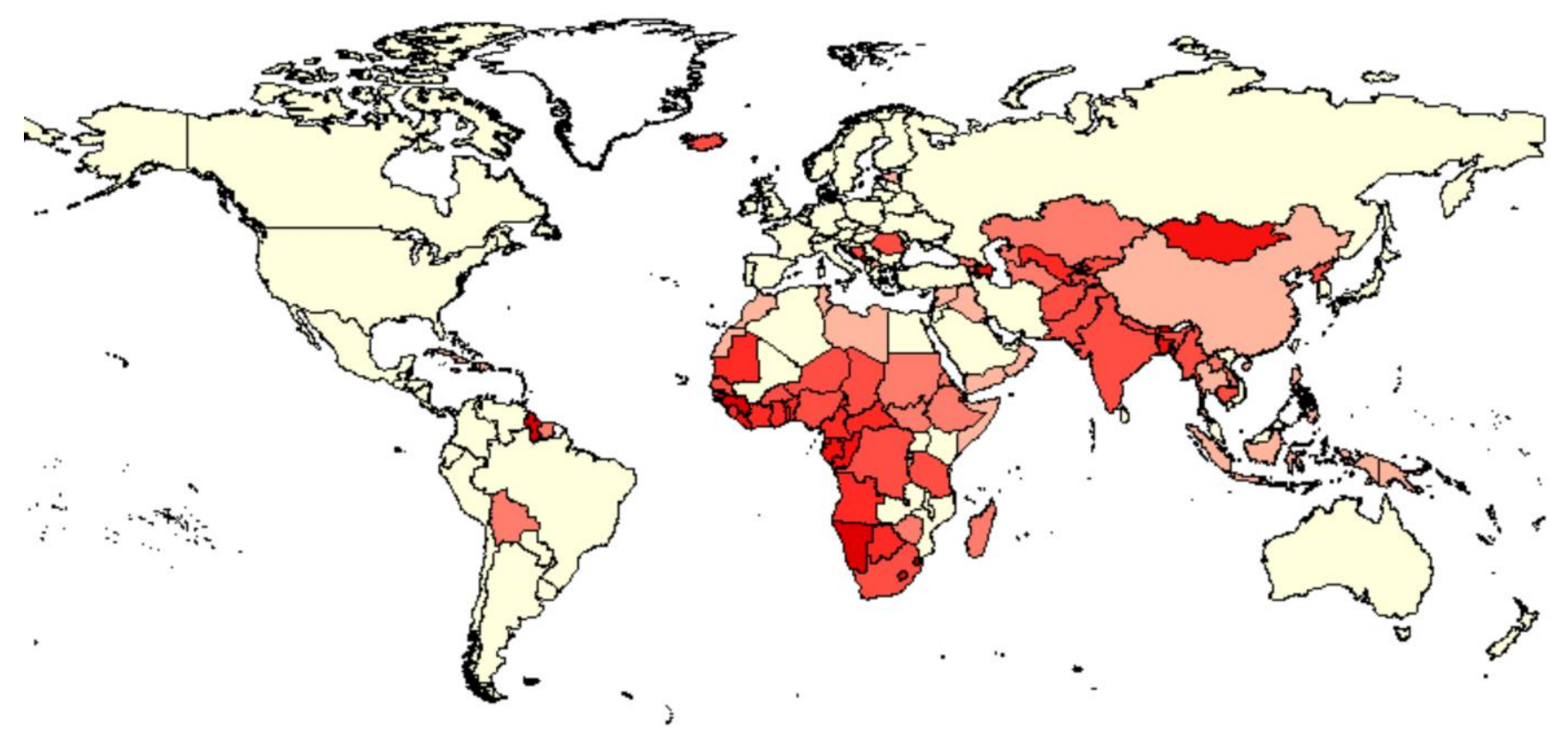

Percent diagnosed

20

30

40 둔.

$50 \square 60 \square 70 \square 80$

This map shows the percentage of children expected to develop cancer each year who are diagnosed. The incidence ratio is calculated by dividing the number of cases reported in a country by the number expected if incidences were the same as those in high-income countries, where non-diagnosis is rare, and the incidence deficit is the incidence ratio subtracted from 100\%. An incidence ratio of $80 \%$ or higher (yellow) is consistent with diagnosis of all cases, since it is within the observed variability across high-income countries. 
Fig. 2 Causes of treatment failure for children with cancer by World Bank income categories for all children with cancer and selected Burkitt lymphoma studies in low-, middle-, and high-income countries

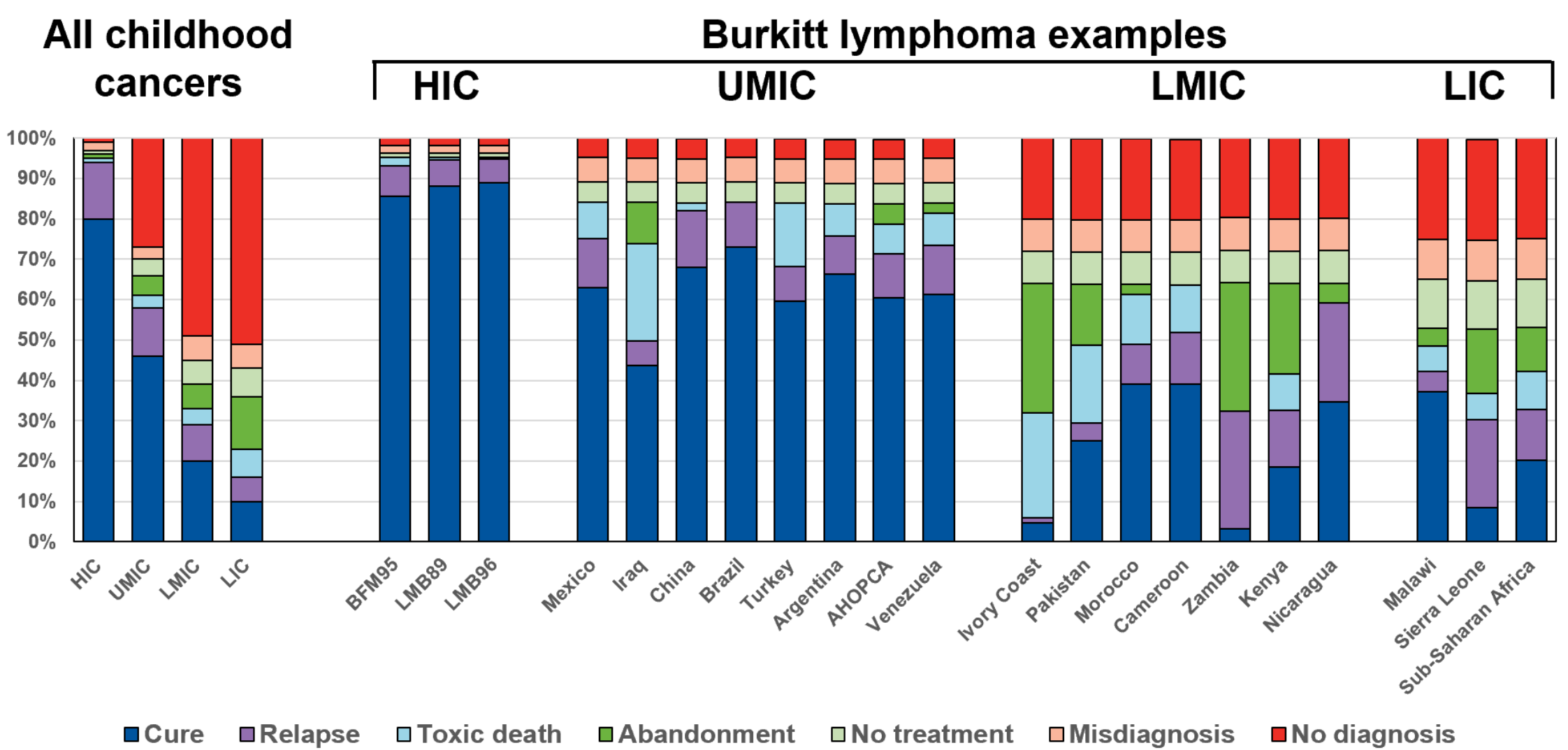

HIC, high-income countries; UMIC, upper-middle-income countries; LMIC, lower-middle-income countries; LIC, low-income countries. Although the causes of treatment failure in HIC differ from those in UMIC, LMIC, and LIC (left section of figure), a variety of published studies of Burkitt lymphoma in each World Bank income group illustrate that even within the same World Bank group, the relative contribution of each cause of treatment failure differs in different settings, even for the same cancer. In the Burkitt lymphoma studies, non-diagnosis, misdiagnosis, and no treatment are estimates, since these are not reported in the clinical trials themselves. Studies specifically addressing misdiagnosis in sub-Saharan African have found rates as high as 18-35\% (26). Used with permission of Scott C. Howard, MD, MSc 
Fig. 3 Cause-specific interventions to reduce treatment failure for children with cancer in low- and middle-income countries

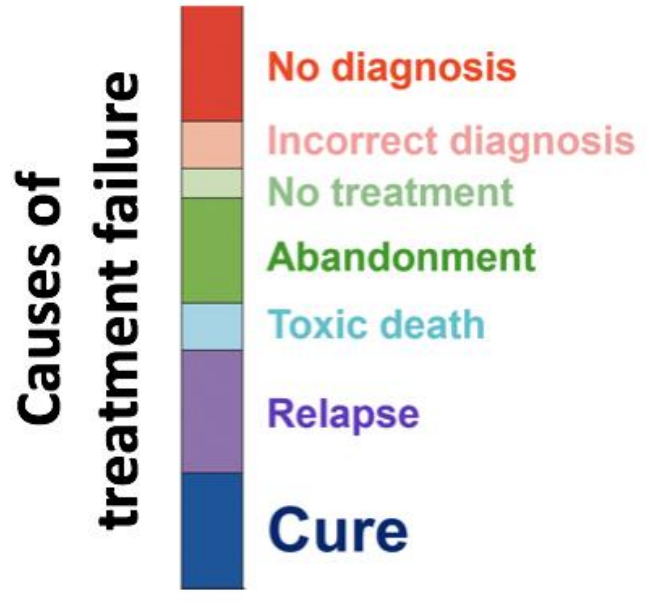

No treatment and abandonment

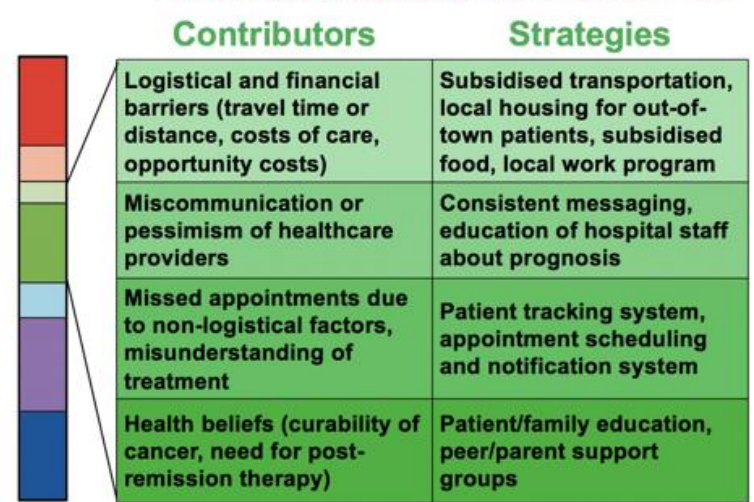

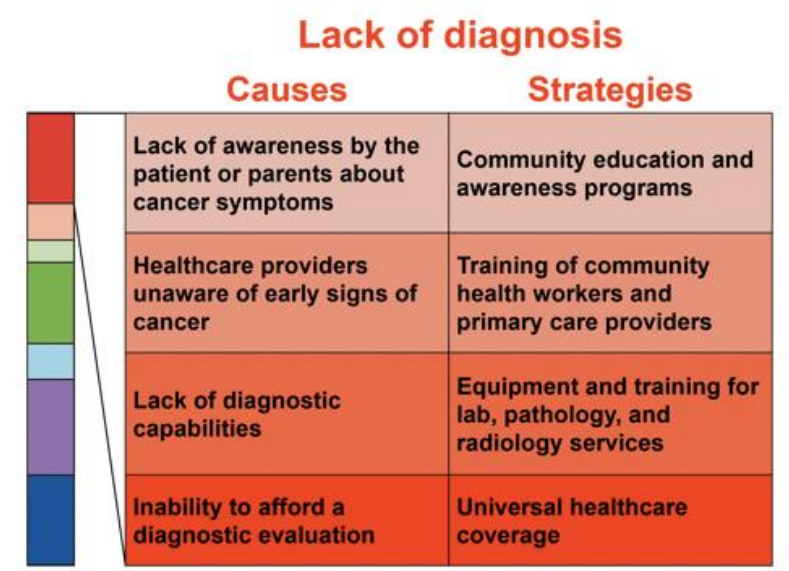

Toxic death

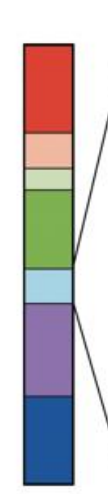

\begin{tabular}{|l|l|}
\multicolumn{1}{c}{ Contributors } & \multicolumn{1}{c}{ Strategies } \\
\hline $\begin{array}{l}\text { Infectious diseases } \\
\text { (bacterial, viral, fungal, } \\
\text { mycobacterial infections) }\end{array}$ & $\begin{array}{l}\text { Hand hygiene, nurse } \\
\text { training, Golden Hour QI, } \\
\text { decrease chemotherapy } \\
\text { intensity, probiotics }\end{array}$ \\
\hline $\begin{array}{l}\text { Haemorrhage (due to } \\
\text { thrombocytopenia, } \\
\text { coagulopathy, and poor } \\
\text { access to blood products) }\end{array}$ & $\begin{array}{l}\text { Blood bank, QI, nurse } \\
\text { training, guest house for } \\
\text { patients living far away }\end{array}$ \\
\hline $\begin{array}{l}\text { Tumor lysis syndrome, } \\
\text { acute kidney injury }\end{array}$ & $\begin{array}{l}\text { Physician and nurse } \\
\text { training, access to } \\
\text { rasburicase and dialysis }\end{array}$ \\
\hline $\begin{array}{l}\text { High-dose methotrexate } \\
\text { and other chemotherapy- } \\
\text { specific toxicities }\end{array}$ & $\begin{array}{l}\text { Physician and nurse } \\
\text { training, Ql, management } \\
\text { of mucositis, pancreatitis }\end{array}$ \\
\hline
\end{tabular}

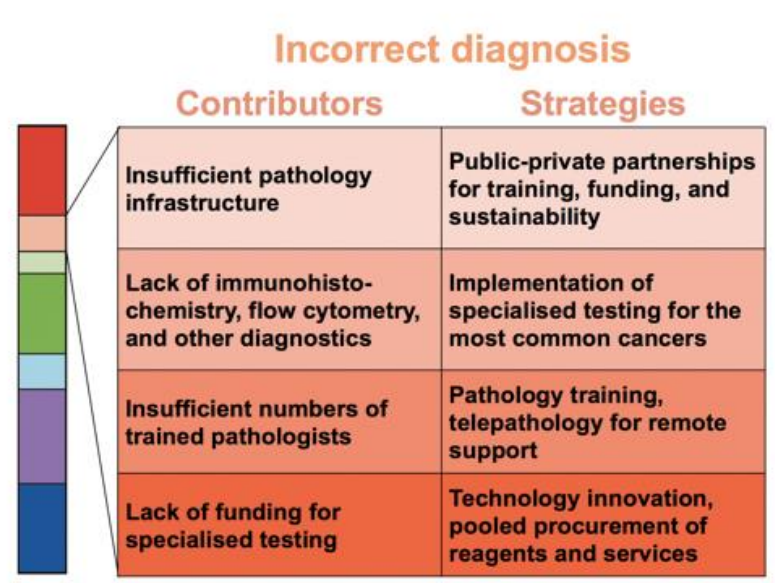

Avoidable relapse

\begin{tabular}{|c|c|}
\hline \multicolumn{2}{|c|}{ 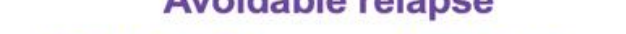 } \\
\hline Contributors & Strategies \\
\hline $\begin{array}{l}\text { Protocols from high- } \\
\text { income countries not } \\
\text { suitable for local } \\
\text { conditions }\end{array}$ & $\begin{array}{l}\text { Deploy locally adapted } \\
\text { protocols, monitor causes } \\
\text { of treatment failure and } \\
\text { adapt }\end{array}$ \\
\hline $\begin{array}{l}\text { Lack of knowledge about } \\
\text { use of adapted protocols, } \\
\text { suboptimal risk } \\
\text { stratification }\end{array}$ & $\begin{array}{l}\text { Personnel training, } \\
\text { monitoring drugs } \\
\text { indicated, prescribed and } \\
\text { administered }\end{array}$ \\
\hline $\begin{array}{l}\text { Gaps in access to } \\
\text { essential chemotherapy }\end{array}$ & $\begin{array}{l}\text { Mitigate drug shortages: } \\
\text { forecast needs, establish } \\
\text { inventory, measure access } \\
\text { and adherence }\end{array}$ \\
\hline $\begin{array}{l}\text { Gaps in adherence to } \\
\text { chemotherapy }\end{array}$ & $\begin{array}{l}\text { Information system/patient } \\
\text { tracking }\end{array}$ \\
\hline
\end{tabular}

Each cause of treatment failure has contributing sub-causes and proven prevention and mitigation strategies. QI, quality improvement. Used with permission of Scott C. Howard, MD, MSc 


\section{References}

1. C. Allemani et al., Global surveillance of trends in cancer survival 2000-14 (CONCORD-3): analysis of individual records for 37513025 patients diagnosed with one of 18 cancers from 322 population-based registries in 71 countries. Lancet 391, 1023-1075 (2018).

2. C. Rodriguez-Galindo et al., Toward the Cure of All Children With Cancer Through Collaborative Efforts: Pediatric Oncology As a Global Challenge. J Clin Oncol 33, 3065-3073 (2015).

3. S. C. Howard et al., Childhood cancer epidemiology in low-income countries. Cancer 112, 461472 (2008).

4. K. Pritchard-Jones et al., Sustaining innovation and improvement in the treatment of childhood cancer: lessons from high-income countries. Lancet Oncol 14, e95-e103 (2013).

5. K. Pritchard-Jones, D. Hargrave, Declining childhood and adolescent cancer mortality: great progress but still much to be done. Cancer 120, 2388-2391 (2014).

6. I. Magrath et al., Paediatric cancer in low-income and middle-income countries. Lancet Oncol 14, e104-116 (2013).

7. World Health Organization, Global Initiative for Childhood Cancer https://www.who.int/cancer/childhood-cancer/en/.

8. E. Steliarova-Foucher et al., International incidence of childhood cancer, 2001-10: a populationbased registry study. Lancet Oncol 18, 719-731 (2017).

9. S. C. Howard et al., The My Child Matters programme: effect of public-private partnerships on paediatric cancer care in low-income and middle-income countries. Lancet Oncol 19, e252-e266 (2018).

10. N. Bhakta et al., Childhood cancer burden: a review of global estimates. Lancet Oncol 20, e42e53 (2019).

11. S. C. Hsu et al., Comparison of treatment outcomes of childhood Hodgkin lymphoma in two US centers and a center in Recife, Brazil. Pediatr. Blood Cancer 49, 139-144 (2007).

12. S. C. Howard et al., Improving outcomes for children with cancer in low-income countries in Latin America: a report on the recent meetings of the Monza International School of Pediatric Hematology/Oncology (MISPHO)-Part I. Pediatr Blood Cancer 48, 364-369 (2007).

13. M. L. Moleti et al., Treatment of children with B-cell non-Hodgkin lymphoma in a low-income country. Pediatr Blood Cancer 56, 560-567 (2011).

14. F. Gaytan-Morales et al., Pediatric mature B-cell NHL, early referral and supportive care problems in a developing country. Hematology 24, 79-83 (2019).

15. C. Cervio et al., Early mortality in children with advanced mature B-cell malignancies in a middleincome country. J Pediatr Hematol Oncol 34, e266-270 (2012).

16. C. Patte et al., Curing Burkitt lymphomas, from France to Africa. Arch Pediatr 22, 65-66 (2015).

17. F. Traore et al., Cyclophosphamide monotherapy in children with Burkitt lymphoma: a study from the French-African Pediatric Oncology Group (GFAOP). Pediatr Blood Cancer 56, 70-76 (2011).

18. T. Israels et al., Malnutrition and neutropenia in children treated for Burkitt lymphoma in Malawi. Pediatr Blood Cancer 53, 47-52 (2009).

19. A. K. Pribnow, R. Ortiz, L. F. Baez, L. Mendieta, S. Luna-Fineman, Effects of malnutrition on treatment-related morbidity and survival of children with cancer in Nicaragua. Pediatr Blood Cancer 64, 10.1002/pbc.26590 (2017).

20. HeadSmart Be Brain Tumour Aware, A new clinical guideline from the Royal College of Paediatrics and Child Health with a national awareness campaign accelerates brain tumor 
diagnosis in UK children--"HeadSmart: Be Brain Tumour Aware". Neuro Oncol 18, 445-454 (2016).

21. C. Leander et al., Impact of an education program on late diagnosis of retinoblastoma in Honduras. Pediatr Blood Cancer 49, 817-819 (2007).

22. UICC, $\mathrm{CCl}$, SIOP, My Child Matters, Childhood cancer 'Signs and Symptoms' campaign: Compilation of campaign materials. https://www.uicc.org/sites/main/files/atoms/files/ChiCaSigns-and-Symptoms Campaign-compilation.pdf.

23. S. Poyiadjis, L. Wainwright, G. Naidu, D. Mackinnon, J. Poole, The Saint Siluan warning signs of cancer in children: impact of education in rural South Africa. Pediatr Blood Cancer 56, 314-316 (2011).

24. Pan American Health Organization, Early Diagnosis of Childhood Cancer. Washington, DC: PAHO, 2014. http://iris.paho.org/xmlui/handle/123456789/34850

25. K. N. Naresh et al., Diagnosis of Burkitt lymphoma using an algorithmic approach--applicable in both resource-poor and resource-rich countries. Br J Haematol 154, 770-776 (2011).

26. J. Orem et al., Agreement between diagnoses of childhood lymphoma assigned in Uganda and by an international reference laboratory. Clin Epidemiol 4, 339-347 (2012).

27. M. D. Ogwang, W. Zhao, L. W. Ayers, S. M. Mbulaiteye, Accuracy of Burkitt lymphoma diagnosis in constrained pathology settings: importance to epidemiology. Arch Pathol Lab Med 135, 445450 (2011).

28. N. S. Parikh et al., SIOP-PODC adapted risk stratification and treatment guidelines:

Recommendations for neuroblastoma in low- and middle-income settings. Pediatr Blood Cancer 62, 1305-1316 (2015).

29. M. Harif et al., Treatment of B-cell lymphoma with LMB modified protocols in Africa--report of the French-African Pediatric Oncology Group (GFAOP). Pediatr Blood Cancer 50, 1138-1142 (2008).

30. T. Santiago et al., Improving Immunohistochemistry Capability for Pediatric Cancer Care in the Central American and Caribbean Region: A Report From the AHOPCA Pathology Working Group. J Glob Oncol 4, 1-9 (2018).

31. T. C. Santiago et al., Improving the histopathologic diagnosis of pediatric malignancies in a lowresource setting by combining focused training and telepathology strategies. Pediatr Blood Cancer 59, 221-225 (2012).

32. S. C. Howard, R. C. Ribeiro, C. H. Pui, Strategies to improve outcomes of children with cancer in low-income countries. Eur. J. Cancer 41, 1584-1587 (2005).

33. A. Samudio et al., Building a National Pediatric Cancer Center and Network in Paraguay: Lessons for Addressing Challenges in a Low-income Country. J Pediatr Hematol Oncol 37, 383-390 (2015).

34. T. Israels et al., Improved outcome at end of treatment in the collaborative Wilms tumour Africa project. Pediatr Blood Cancer 65, e26945 (2018).

35. V. Paintsil et al., The Collaborative Wilms Tumour Africa Project; baseline evaluation of Wilms tumour treatment and outcome in eight institutes in sub-Saharan Africa. Eur J Cancer 51, 84-91 (2015).

36. G. Acquatella et al., Outcome of children with B cell lymphoma in Venezuela with the LMB-89 protocol. Pediatr. Blood Cancer 43, 580-586 (2004).

37. N. Ahmad, A. Zaidi, F. Badar, A. U. Maaz, M. S. Akram, Clinical characteristics and outcome analysis of pediatric B-cell non-Hodgkin's lymphoma. Experience with FAB-LMB 96 and UKCCSG B-cell NHL guidelines in a developing country. Asia Pac J Clin Oncol 6, 49-56 (2010).

38. S. C. Howard et al., Establishment of a pediatric oncology program and outcomes of childhood acute lymphoblastic leukemia in a resource-poor area. JAMA 291, 2471-2475 (2004). 
39. F. Ceppi et al., Supportive medical care for children with acute lymphoblastic leukemia in lowand middle-income countries. Expert Rev Hematol 8, 613-626 (2015).

40. M. S. Cairo, B. Coiffier, A. Reiter, A. Younes, T. L. S. E. Panel, Recommendations for the evaluation of risk and prophylaxis of tumour lysis syndrome (TLS) in adults and children with malignant diseases: an expert TLS panel consensus. Br J Haematol 149, 578-586 (2010).

41. E. J. Ladas et al., A Framework for Adapted Nutritional Therapy for Children With Cancer in Lowand Middle-Income Countries: A Report From the SIOP PODC Nutrition Working Group. Pediatr Blood Cancer 63, 1339-1348 (2016).

42. A. Agulnik et al., Validation of a pediatric early warning system for hospitalized pediatric oncology patients in a resource-limited setting. Cancer 123, 4903-4913 (2017).

43. A. Agulnik et al., Improved outcomes after successful implementation of a pediatric early warning system (PEWS) in a resource-limited pediatric oncology hospital. Cancer 123, 2965-2974 (2017).

44. C. G. Sanchez La Rosa et al., Improved Outcome and Decreased Morbidity and Mortality Rates of B-Cell Malignancies with Less Intensive Chemotherapy Induction: Experience in a Single Institution. Blood 128, 1858-1858 (2016).

45. A. O. Peña-Hernandez, R. Garrido, C.; Gomez-Garcia, W.; ,Fuentes Alabi, S.; Martinez, R; Metzger, M.; Chantada, G.; Ribeiro RC, Treatment results of a strategy for the management of pediatric non-Hodgkin lymphoma in Central America: A report of the Association of Pediatric Hematology/Oncology of Central America (AHOPCA). Pediatr Blood Cancer Jan 24:e27621. doi: 10.1002/pbc.27621., (2019).

46. S. C. Howard et al., Protocol-based treatment for children with cancer in low income countries in Latin America: a report on the recent meetings of the Monza International School of Pediatric Hematology/Oncology (MISPHO)--part II. Pediatr Blood Cancer 48, 486-490 (2007).

47. A. Davidson, S. C. Howard, Delivering modern anticancer therapies in low- and middle-income settings: We can be evidence based. Pediatr Blood Cancer, e27347 (2018).

48. K. Vaishnavi, D. Bansal, A. Trehan, R. Jain, S. V. Attri, Improving the safety of high-dose methotrexate for children with hematologic cancers in settings without access to MTX levels using extended hydration and additional leucovorin. Pediatr Blood Cancer 65, e27241 (2018).

49. C. E. Klumb et al., Treatment of children with B-cell non-Hodgkin's lymphoma in developing countries: the experience of a single center in Brazil. J Pediatr Hematol Oncol 26, 462-468 (2004).

50. X. F. Sun et al., Improved treatment outcome in Chinese children and adolescents with Burkitt's lymphoma and large cell lymphoma by using the modified B-non-Hodgkin's lymphoma-BerlinFrankfurt-Munster-90 protocol. Eur J Haematol 77, 365-371 (2006).

51. L. Roth et al., Expanding global access to essential medicines: investment priorities for sustainably strengthening medical product regulatory systems. Global Health 14, 102 (2018).

52. S. K. Perehudoff, N. V. Alexandrov, H. V. Hogerzeil, Access to essential medicines in 195 countries: A human rights approach to sustainable development. Glob Public Health, 1-14 (2018).

53. P. Khetrapal Singh, P. Travis, Accelerating access to essential medicines in the WHO South-East Asia Region: opportunities for greater engagement and better evidence. WHO South East Asia J Public Health 7, 59-61 (2018).

54. P. Cohen et al., Global Access to Essential Medicines for Childhood Cancer: A Cross-Sectional Survey. Journal of Global Oncology, 1-11 (2018).

55. P. Friedrich et al., Determinants of Treatment Abandonment in Childhood Cancer: Results from a Global Survey. PLoS One 11, e0163090 (2016). 
56. J. Parkes et al., Recommendations for the treatment of children with radiotherapy in low- and middle-income countries (LMIC): A position paper from the Pediatric Radiation Oncology Society (PROS-LMIC) and Pediatric Oncology in Developing Countries (PODC) working groups of the International Society of Pediatric Oncology (SIOP). Pediatr Blood Cancer 64 Suppl 5, doi: 10.1002/pbc.26903 (2017).

57. S. C. Howard et al., A framework to develop adapted treatment regimens to manage pediatric cancer in low- and middle-income countries: The Pediatric Oncology in Developing Countries (PODC) Committee of the International Pediatric Oncology Society (SIOP). Pediatr Blood Cancer 64 Suppl 5, https://doi.org/10.1002/pbc.26879 (2017).

58. R. S. Arora, J. M. Challinor, S. C. Howard, T. Israels, Improving Care for Children With Cancer in Low- and Middle-Income Countries--a SIOP PODC Initiative. Pediatr Blood Cancer 63, 387-391 (2016).

59. S. Mostert et al., Abandonment of treatment for childhood cancer: position statement of a SIOP PODC Working Group. Lancet Oncol 12, 719-720 (2011).

60. S. Mostert et al., Hospital detention practices: statement of a global taskforce. Lancet 386, 649 (2015).

61. M. L. Metzger et al., Outcome of childhood acute lymphoblastic leukaemia in resource-poor countries. Lancet 362, 706-708 (2003).

62. P. Friedrich et al., Magnitude of Treatment Abandonment in Childhood Cancer. PLoS One 10, e0135230 (2015).

63. S. Gupta et al., The magnitude and predictors of abandonment of therapy in paediatric acute leukaemia in middle-income countries: a systematic review and meta-analysis. Eur J Cancer 49, 2555-2564 (2013).

64. J. D. De Boer, T. N. Boellaard, S. Parkinson, E. Blanchard, H. A. Heij, Patient compliance in the treatment of Burkitt's lymphoma in rural Zambia: a retrospective study on 80 Burkitt's lymphoma patients in Katete, Zambia. Afr J Paediatr Surg 6, 3-6 (2009).

65. J. J. Yao et al., Childhood cancer in Cote d'Ivoire, 1995 - 2004: challenges and hopes. S Afr Med J 103, 113-115 (2012).

66. H. A. Martijn et al., Influence of health insurance status on paediatric non-Hodgkin's lymphoma treatment in Kenya. BMJ Paediatr Open 1, e000149 (2017).

67. F. Ceppi et al., Anaplastic Large Cell Lymphoma in Central America: A Report From the Central American Association of Pediatric Hematology Oncology (AHOPCA). Pediatr Blood Cancer 63, 7882 (2016).

68. E. Alvarez et al., Improvement in treatment abandonment in pediatric patients with cancer in Guatemala. Pediatr Blood Cancer 64, doi: 10.1002/pbc.26560 (2017).

69. S. Mostert et al., Two overlooked contributors to abandonment of childhood cancer treatment in Kenya: parents' social network and experiences with hospital retention policies.

Psychooncology 23, 700-707 (2014).

70. M. San Roman et al., Burkitt's lymphoma treatment in a rural hospital in Sierra Leone. Trans $R$ Soc Trop Med Hyg 107, 653-659 (2013).

71. C. Salaverria et al., Interventions targeting absences increase adherence and reduce abandonment of childhood cancer treatment in El Salvador. Pediatr Blood Cancer 62, 1609-1615 (2015).

72. World Health Organization, National Cancer Control Programmes https://www.who.int/cancer/nccp/en/.

73. R. C. Ribeiro, F. Antillon, F. Pedrosa, C. H. Pui, Global Pediatric Oncology: Lessons From Partnerships Between High-Income Countries and Low- to Mid-Income Countries. J Clin Oncol 34, 53-61 (2016). 
74. M. S. Weaver et al., A practical approach to reporting treatment abandonment in pediatric chronic conditions. Pediatr Blood Cancer 62, 565-570 (2015).

75. M. S. Weaver, A. J. Yao, L. A. Renner, M. Harif, C. G. Lam, The prioritisation of paediatrics and palliative care in cancer control plans in Africa. Br J Cancer 112, 1845-1856 (2015).

76. Kenya Ministry of Health, National Cancer Control Strategy 2017 - 2022, Nairobi, June 2017. www.health.go.ke

77. Ministry of Social Affairs and Public Health Belgium, National Cancer Plan 2008-2010, March 2008. http://www.epaac.eu/from heidi wiki/Belgium National Cancer Plan 2008-

2010 English.pdf.

78. NHS National Cancer Survivorship Initiative, Building the evidence: Developing the winning principles for children and young people.

https://webarchive.nationalarchives.gov.uk/20130513172528/http://www.improvement.nhs.uk /documents/CYP Building the Evidence.pdf.

79. World Health Organization, WHO Model List of Essential Medicines. https://www.who.int/medicines/publications/essentialmedicines/en/.

80. J. Robertson, R. Barr, L. N. Shulman, G. B. Forte, N. Magrini, Essential medicines for cancer: WHO recommendations and national priorities. Bull World Health Organ 94, 735-742 (2016).

81. US Food and Drug Administration, https://www.fda.gov/Drugs/.

82. S. Blackman, In short supply: tackling the drug shortage crisis. Pharm Tech 28 October 2012.

https://www.pharmaceutical-technology.com/features/featureshort-supply-tackling-drug-shortagecrisis/.

83. P. P. Zenatti et al., Low Bioavailability and High Immunogenicity of a New Brand of E. colilAsparaginase with Active Host Contaminating Proteins. EBioMedicine 30, 158-166 (2018).

84. D. K. Cecconello et al., Monitoring asparaginase activity in middle-income countries. Lancet Oncol 19, 1149-1150 (2018).

85. C. E. DeSantis, J. L. Kramer, A. Jemal, The burden of rare cancers in the United States. CA Cancer J Clin 67, 261-272 (2017).

86. C. L. Freeman, L. Sehn, Anti-CD20 Directed Therapy of B Cell Lymphomas: Are New Agents Really Better? Curr Oncol Rep 20, 103 (2018).

87. J. M. L. Casan, J. Wong, M. J. Northcott, S. Opat, Anti-CD20 monoclonal antibodies: reviewing a revolution. Hum Vaccin Immunother, 1-22 (2018).

88. N. Gaspar et al., Joint adolescent-adult early phase clinical trials to improve access to new drugs for adolescents with cancer: proposals from the multi-stakeholder platform-ACCELERATE. Ann Oncol 29, 766-771 (2018).

89. H. K. Lyerly et al., Global Development of Anticancer Therapies for Rare Cancers, Pediatric Cancers, and Molecular Subtypes of Common Cancers. J Glob Oncol, 1-11 (2018).

90. Accelerate Platform, (https://www.accelerate-platform.eu/)

91. W. Woessmann et al., The impact of the methotrexate administration schedule and dose in the treatment of children and adolescents with B-cell neoplasms: a report of the BFM Group Study NHL-BFM95. Blood 105, 948-958 (2005).

92. N. Amayiri et al., Video-Teleconferencing in Pediatric Neuro-Oncology: Ten Years of Experience. J Glob Oncol, 1-7 (2018).

93. I. Qaddoumi, E. Bouffet, Supplementation of a successful pediatric neuro-oncology telemedicine-based twinning program by e-mails. Telemedicine journal and e-health : the official journal of the American Telemedicine Association 15, 975-982 (2009).

94. I. Qaddoumi et al., Diffuse pontine glioma in Jordan and impact of up-front prognosis disclosure with parents and families. Journal of child neurology 24, 460-465 (2009). 
95. I. Qaddoumi et al., Impact of telemedicine on pediatric neuro-oncology in a developing country: the Jordanian-Canadian experience. Pediatr Blood Cancer 48, 39-43 (2007).

96. I. Qaddoumi et al., Closing the survival gap: implementation of medulloblastoma protocols in a low-income country through a twinning program. International journal of cancer 122, 1203-1206 (2008).

97. N. Amayiri et al., High frequency of mismatch repair deficiency among pediatric high grade gliomas in Jordan. Int J Cancer 138, 380-385 (2016).

98. I. F. Pollack et al., Mismatch repair deficiency is an uncommon mechanism of alkylator resistance in pediatric malignant gliomas: a report from the Children's Oncology Group. Pediatr Blood Cancer 55, 1066-1071 (2010).

99. E. Bouffet et al., Immune Checkpoint Inhibition for Hypermutant Glioblastoma Multiforme Resulting From Germline Biallelic Mismatch Repair Deficiency. J Clin Oncol 34, 2206-2211 (2016).

100. M. AlHarbi et al., Durable Response to Nivolumab in a Pediatric Patient with Refractory Glioblastoma and Constitutional Biallelic Mismatch Repair Deficiency. Oncologist 23, 1401-1406 (2018).

101. T. Israels, E. M. Molyneux, S. A.-P. C. W. T. P. Group, Lessons learned from a multicentre clinical trial in Africa. Nat Rev Clin Oncol, (2018).

102. A. E. J. Yeoh et al., Intensifying Treatment of Childhood B-Lymphoblastic Leukemia With IKZF1 Deletion Reduces Relapse and Improves Overall Survival: Results of Malaysia-Singapore ALL 2010 Study. J Clin Oncol 36, 2726-2735 (2018).

103. T. Israels et al., Survival of children with a Wilms tumor in Blantyre, Malawi. Pediatr Hematol Oncol 35, 196-202 (2018).

104. T. Israels, E. M. Molyneux, Siop Africa-PODC Collaborative Wilms Tumour Project Group, Lessons learned from a multicentre clinical trial in Africa. Nat Rev Clin Oncol 10.1038/s41571-018-01210, (2018).

105. D. E. Bloom, A. Khoury, R. Subbaraman, The promise and peril of universal health care. Science 361, (2018).

106. Alma-Ata 1978 Primary Health Care, Report of the International Conference on Primary Health Care Alma-Ata, USSR

https://www.unicef.org/about/history/files/Alma Ata conference 1978 report.pdf Last accessed December 21, 2018.

107. M. Jankovic et al., Long-term survivors of childhood cancer: cure and care-the Erice Statement (2006) revised after 10 years (2016). J Cancer Surviv 12, 647-650 (2018).

108. G. Chantada, C. G. Lam, S. C. Howard, Optimizing outcomes for children with non-Hodgkin lymphoma in low- and middle-income countries by early correct diagnosis, reducing toxic death, and preventing abandonment. BrJ Haematol DOI:10.1111/bjh.15785, (2019).

109. United Nations Population Statistics, http://www.un.org/en/development/desa/population/publications/database/index.shtml. 


\section{Other Academic Affiliations:}

C.G.L. is Co-Director of the World Health Organization Collaborating Centre for Childhood Cancer.

S.C.H. is Secretary-General, International Society of Paediatric Oncology.

E.B. is President, International Society of Paediatric Oncology.

K.P. J. (in addition to UCL) is affiliated with Centre for Cancer Outcomes, University College London Hospitals (UCLH), London, UK; Cancer Programme Director, UCL Partners Academic

Health Science Network, London, UK ; President-elect, International Society of Paediatric Oncology.

Funding: include complete funding information.

C.G.L. is funded at St. Jude by the American Lebanese Syrian Associated Charities.

E.B. is receiving funding from Bristol Myers Squibb for an investigator-initiated study of immunotherapy (NCT02992964).

K.P.J. is funded in part by the National Institute of Health Research Biomedical Research Centre at Great Ormond Street Hospital.

Competing interests: The authors have no competing interests. 